\title{
Switchable Josephson current in junctions with spin-orbit coupling
}

\author{
B. Bujnowski, ${ }^{1, *}$ R. Biele, ${ }^{2,3}$ and F. S. Bergeret ${ }^{1,4, \uparrow}$ \\ ${ }^{1}$ Donostia International Physics Center (DIPC) - Manuel de Lardizabal 5, E-20018 San Sebastián, Spain \\ ${ }^{2}$ Max Bergmann Center of Biomaterials, TU Dresden, 01062 Dresden, Germany \\ ${ }^{3}$ Institute for Materials Science, TU Dresden, 01062 Dresden, Germany \\ ${ }^{4}$ Centro de Física de Materiales (CFM-MPC) Centro Mixto CSIC-UPV/EHU - 20018 Donostia-San Sebastian, Basque Country, Spain
}

(Received 4 September 2019; revised manuscript received 21 October 2019; published 27 December 2019)

\begin{abstract}
We study the Josephson current in two types of lateral junctions with spin-orbit coupling and an exchange field. The first system (type 1 junction) consists of superconductors with heavy metal interlayers linked by a ferromagnetic bridge, such that the spin-orbit coupling is finite only at the superconductor/heavy metal interface. In the second type (type 2) of system we assume that the spin-orbit coupling is finite in the bridge region. The length of both junctions is larger than the magnetic decay length such that the Josephson current is carried uniquely by the long-range triplet component of the condensate. The latter is generated by the spin-orbit coupling via two mechanisms, spin precession and inhomogeneous spin relaxation. We show that the current can be controlled by rotating the magnetization of the bridge or by tuning the strength of the spin-orbit coupling in type 2 junctions and also discuss how the ground state of the junction can be tuned from a 0 to a $\pi$ phase difference between the superconducting electrodes. In leading order in the spin-orbit coupling, the spin precession dominates the behavior of the triplet component and both junctions behave similarly. However, when spin relaxation effects are included the type 2 junction offers a wider parameter range in which $0-\pi$ transitions take place.
\end{abstract}

DOI: 10.1103/PhysRevB.100.224518

\section{INTRODUCTION}

The interplay between superconductivity and ferromagnetism leads to triplet superconducting correlations [1-4]. The simplest setup for the generation of a triplet component is a superconductor (S)-ferromagnet $(\mathrm{F})$ heterostructure with a homogeneous exchange field. The superconducting singlet Cooper pairs can penetrate the ferromagnet, and due to the local exchange field are partially converted into triplet pairs with the total spin projection zero with respect to the local exchange field. Oscillations of the triplet correlations in the $\mathrm{F}$ region lead to the well understood effect of the sign reversal of the critical current, the so-called $0-\pi$ transition [5-9]. In a diffusive monodomain $F$, both singlet and triplet correlations decay on the magnetic length scale $\xi_{h}=\sqrt{D / h}$, where $h$ is the magnitude of the exchange field and $D$ is the diffusion constant. For conventional Ss and typical exchange field strengths, $\xi_{h}$ is much shorter than the thermal length scale of decay $\xi_{\omega} \approx \sqrt{D / T}$ in a nonmagnetic system. On the other hand, triplet components with nonzero spin projection are not affected by its pair breaking effect and would decay over a length scale comparable to $\xi_{\omega}$. Such long-range triplet components (LRTC) can be generated due to inhomogeneities of the exchange field $[1,2,4]$ or due to the presence of spin-orbit coupling (SOC) and a homogeneous exchange field $[10,11]$.

\footnotetext{
*bogusz.bujnowski@gmail.com

${ }^{\dagger}$ fs.bergeret@csic.es
}

The prediction of LRTC in S/F hybrid structures has stimulated multiple experimental works [12-22]. More recently, transverse vertical heterostructures with in-plane magnetic fields and SOC materials have been experimentally explored but the long-range correlations due to SOC have not been observed [23-25]. In accordance with previous theoretical works $[10,11]$ in vertical multilayered SFS junctions the condition for the generation of a LRTC is quite restrictive. More suitable for the observation of LRTC induced by the SOC are lateral structures where currents have also a component flowing in the direction parallel to the hybrid interface [26-28].

In this work we present a study of the Josephson current in lateral geometries with SOC of Rashba and Dresselhaus type and how to control it via external fields in the diffusive regime. We focus on two types of junctions: One consists of two superconducting electrodes on top of a ferromagnetic film, see Fig. 1(a). Between the two materials we assume there is an interlayer with a finite SOC. Hereafter we refer to this junction as a type 1 junction. The junction of type 2, Fig. 1(b), consists of a similar lateral geometry, but the SOC is finite in the bridge region. Whereas type 1 junctions may correspond to junctions with a heavy metal interlayer, type 2 junctions describe, for example, a lateral Josephson junction made of a 2D electron gas in the presence of a Zeeman field. We assume that in both junctions the distance between the superconductor electrodes is larger than the magnetic decay length, such that the Josephson current is only carried by LRTC. The latter is generated by the SOC via two mechanisms: spin precession and inhomogeneous spin-relaxation, and the current strongly depends on the direction of the exchange or Zeeman field. In addition, in type 2 junctions the Josephson current can also be 


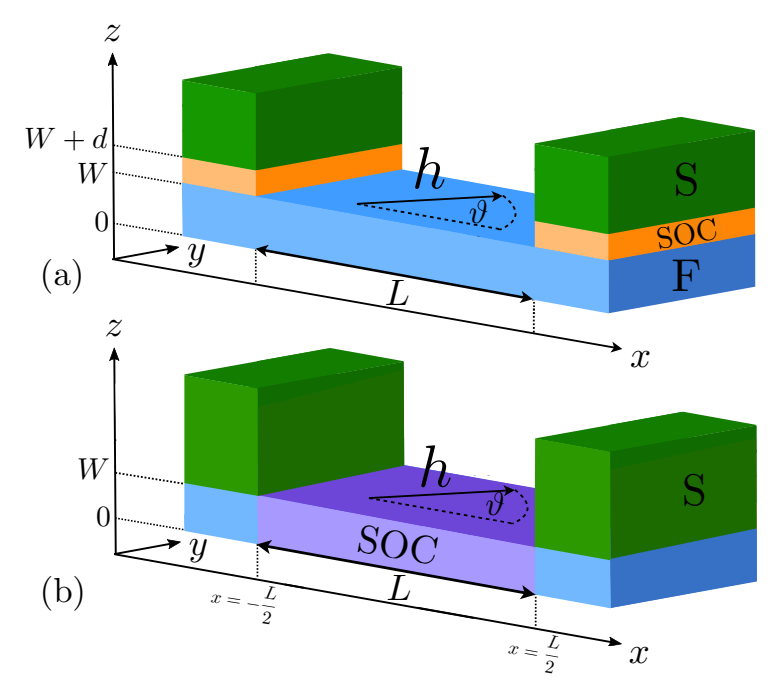

FIG. 1. Schematic view of the two junction types considered in the text. (a) The junction of type 1 consists of two superconductors contacted to a ferromagnet $(\mathrm{F})$ via a material with strong SOC. The magnetization of $\mathrm{F}$, and hence the exchange field $\mathbf{h}$, lies in the $x-y$ plane. (b) For the junction of type 2 the bridge region connecting the two superconductors has a sizable SOC.

tuned by a voltage gate that controls the strength of the Rashba SOC.

We focus on the control of possible $0-\pi$ transitions. With the help of an analytical solution for type 1 junction in the case of small SOC, we first show that in leading order the LRTC is generated only by the spin-precession term and the junction remains in the 0 state independently of the direction of the exchange field. The next leading order contribution to the current is due to the inhomogeneous spin relaxation with a negative sign, such that for certain directions of the exchange field the junction can switch to the $\pi$ state. In junctions of type 1 this only occurs if both the Rashba and Dresselhaus SOC are finite. In a second part we present numeric calculations of the current for arbitrary SOC strength that confirm these findings. In addition these calculations reveal that type 2 junctions allow for $0-\pi$ transitions in a wider range of SOC parameters. Specifically the transition can be induced by a pure Rashba or Dresselhaus SOC by changing their strengths. This is a new possibility to induce $0-\pi$ transition by tuning the Rashba SOC strength, which is experimentally achievable by gating the SOC active material. Besides the interesting applications of such lateral junctions as $0-\pi$ switchers, they can also be used to detect the LRTC by measuring the changes of the Josephson current as a function of the direction of the applied field or magnetization in a single junction.

The work is organized as follows: In Sec. II we present the basic equations describing diffusive Josephson junctions and we adapt these equations to the lateral junctions type 1 and 2. In Sec. III we derive the analytical expression for the Josephson current in junction type 1 perturbatively, up to second order in the SOC parameter for semi-infinite leads. In Sec. IV we present numerical results for the Josephson current for both types of junctions and compare them to the analytical results. Conclusions are given in Sec. V.

\section{BASIC EQUATIONS FOR DIFFUSIVE JOSEPHSON JUNCTIONS WITH SOC}

We consider two spatially separated superconducting electrodes on top of a nonsuperconducting material with either an intrinsic exchange field, as in a ferromagnet, or a Zeeman field induced by an external magnetic field. We distinguish two different types of junctions: one with SOC active layers just below the superconductors, Fig. 1(a), that we refer to as junction type 1 . The other junction with SOC in the bridge region is referred to as junction type 2 and is shown in Fig. 1(b).

We assume that the proximity effect, i.e., the induced superconducting correlations in the bridge, is weak and that the system is in the diffusive regime. In this case spectral and transport properties of the junction can be accurately described by the linearized Usadel equation [29] generalized to linear in momentum SOC. This equation provides the spatial dependence of the induced superconducting correlations in the nonsuperconducting region which is described in terms of the anomalous Green's function $\hat{f}[10,11][30]$ :

$$
D \tilde{\nabla}_{k}^{2} \hat{f}+2\left|\omega_{n}\right| \hat{f}-i \operatorname{sign}\left(\omega_{n}\right)\{\hat{h}, \hat{f}\}=0 .
$$

Here $D$ is the diffusion constant, $\omega_{n}$ is the Matsubara frequency, $\tilde{\nabla}_{k}=\partial_{k}-i\left[\hat{\mathcal{A}}_{k}, \ldots\right]$ is the covariant derivative with the $\mathrm{SU}(2)$ vector potential, $\hat{\mathcal{A}}_{k}=\frac{\hat{\sigma}^{a}}{2} \mathcal{A}_{k}^{a}$, describing the SOC, and $\hat{h}=\hat{\sigma}^{a} h^{a}$ is the exchange field. Symbols with a ${ }^{\wedge}$ stand for operators in spin space and $\hat{\sigma}^{a}$ are the Pauli matrices. We use the Einstein summation convention and sum over repeated indices. The general form of the condensate function in spin space is

$$
\hat{f}=f_{s} \hat{1}+f_{t}^{a} \hat{\sigma}^{a}
$$

where $f_{s}$ is the singlet component and $f_{t}^{a}$ are the triplet components. In our representation the short (long)-range triplet component corresponds to the component parallel (orthogonal) to the exchange or Zeeman field.

In order to describe hybrid interfaces between the superconductor and a substrate one needs boundary conditions for the Green's functions. We use here the Kupriyanov-Lukichev ones [31] generalized for materials with SOC. In its linearized form at an $S / X$ interface they read $[10,11]$ :

$$
\mathcal{N}_{i}\left[\tilde{\nabla}_{i} \hat{f}\right]_{S / X}=-\gamma f_{B C S} \hat{1},
$$

where $X$ denotes any nonsuperconductor material. Here $\mathcal{N}_{i}$ is the $i$ th component of the interface normal, $\gamma$ is the interface transparency, $f_{B C S}=\frac{\Delta e^{i \varphi_{i}}}{\sqrt{\Delta^{2}-\omega_{n}^{2}}}$ is the anomalous Green's function in the bulk $S$ region with the amplitude of the superconducting order parameter $\Delta$ and its phase $\varphi_{i}$ in the $i$ th electrode. At the interface with vacuum $(\mathrm{V})$ no current flows and the boundary condition reads:

$$
\mathcal{N}_{i}\left[\tilde{\nabla}_{i} \hat{f}\right]_{X / V}=0
$$

Below we determine the Josephson current density in the bridge region for the two setups depicted in Fig. 1. It can be 
expressed as [32]:

$$
j=i e \pi N_{0} D T \sum_{\omega_{n}} \operatorname{Tr}\{\hat{f} \tilde{\nabla} \hat{\bar{f}}-\hat{\tilde{f}} \tilde{\nabla} \hat{f}\}
$$

where $N_{0}$ is the density of states and $\hat{\bar{f}}=\hat{\sigma}^{y} \hat{f}^{*} \hat{\sigma}^{y}$.

We now rewrite Eqs. (1) and (3) for the specific case of junction of type 1 and 2. Both junctions are assumed to be translational invariant in the $y$ direction. The order parameter is a steplike function along the $x$ direction, with amplitude $\Delta$ at the $\mathrm{S}$ electrodes and zero at the bridge. We denote the phase difference between the superconductors with $\varphi$, such that

$$
\Delta(x, z)=\Theta(z-(W+d)) \Theta(|x|-L / 2) \Delta e^{i \frac{\varphi}{2} \operatorname{sign}(x)} .
$$

The SOC fields are finite only in the SOC layers thus for the junction type 1 [Fig. 1(a)]

$$
\mathcal{A}_{k}^{a}(x, z)=\Theta(W+d-z) \Theta(z-W) \Theta(|x|-L / 2) \mathcal{A}_{k}^{a}
$$

and for the junction type 2 with the SOC in the bridge region [Fig. 1(b)]

$$
\mathcal{A}_{k}^{a}(x, z)=\Theta(W-z) \Theta(z-W) \Theta(L / 2-|x|) \mathcal{A}_{k}^{a}
$$

with constant $\mathcal{A}_{k}^{a}$. We restrict ourselves to SOC of the Rashba and Dresselhaus type defined by the following vector potential: $\hat{\mathcal{A}}_{x}=\beta / 2 \hat{\sigma}^{x}-\alpha / 2 \hat{\sigma}^{y}$ and $\hat{\mathcal{A}}_{y}=\alpha / 2 \hat{\sigma}^{x}-\beta / 2 \hat{\sigma}^{y}$. Rashba SOC corresponds to terms proportional to $\alpha$, while Dresselhaus SOC corresponds to terms proportional to $\beta$. For both junctions the exchange field has only finite components in the $x-y$ plane and is present in the region $\mathrm{F}$,

$$
\hat{h}(x, z)=h\left(\cos \vartheta \hat{\sigma}^{x}+\sin \vartheta \hat{\sigma}^{y}\right) \Theta(z) \Theta(W-z),
$$

where $h=\sqrt{h^{a} h^{a}}$.

To distinguish components that are parallel and perpendicular to the exchange field, i.e., short- and long-range components, it is convenient to rotate Eqs. (1) and (3) by the unitary transformation $\mathcal{U}=e^{i \hat{\sigma}^{x \frac{\vartheta}{2}}}$. After the rotation the exchange field is fixed along the $x$ axis,

$$
\mathcal{U} \hat{\mathcal{U}^{\dagger}}=h \hat{\sigma}^{x} .
$$

Thus in our notation the LRTC are those polarized in the $y$ and $z$ direction. Assuming for simplicity that the thickness $d$ of the SOC interlayers (if present) and the bridge $W$ is smaller than the typical length on which $\hat{f}$ changes, i.e., $d \ll \xi_{\omega}$ and $W \ll \xi_{h}$, we can integrate the Usadel equation along the $z$ direction [11]. This reduces the initial two-dimensional problem to an effective one-dimensional one. Thicker layers will require the solution of a $2 \mathrm{D}$ problem, but qualitatively our results should be valid also in that case.

Here we illustrate how the $z$ integration is carried out. Besides the first term in Eq. (1), all other terms do not contain a spatial derivative in the $z$ direction and therefore the integration results simply in the averaged value of $f$. Integration of the first term of Eq. (1) leads to

$$
\begin{aligned}
\int_{0}^{W+d} \tilde{\nabla}_{k}^{2} \hat{f} d z= & \int_{0}^{W+d} d z\left(\left(\partial_{x}^{2}+\partial_{z}^{2}\right) \hat{f}\right. \\
& \left.-2 i\left[\hat{A}_{x}, \partial_{x} \hat{f}\right]-\left[\hat{A}_{k},\left[\hat{A}_{k}, \hat{f}\right]\right]\right) \\
\approx & \gamma f_{B C S}+(W+d) \partial_{x}^{2} \hat{f}-2 i d\left[\hat{A}_{x}, \partial_{x} \hat{f}\right] \\
& -d\left[\hat{A}_{k},\left[\hat{A}_{k}, \hat{f}\right]\right] .
\end{aligned}
$$

In the first step we have used the translational invariance in the $y$ direction and our choice of the SOC, which is stepwise constant. In the second step we use the continuity of $\hat{f}$ in the $z$ direction and the boundary condition Eq. (3) at the interface at $z=W+d$ as well as the boundary condition with the vacuum, Eq. (4). The $z$ integration causes an averaging of the couplings that differs for the two junction types. We therefore present the final equations separately.

\section{A. Usadel equations for type 1 lateral junction}

After performing the $z$ integration, and the rotation of Eq. (1), the resulting system of equations for the rotated anomalous Green's function $\hat{\tilde{f}}=\mathcal{U} \hat{f} \mathcal{U}^{\dagger}$ is

$D\left[\partial_{x}^{2} \tilde{f}_{s}\right]-2\left|\omega_{n}\right| \tilde{f}_{s}-2 i \operatorname{sign}\left(\omega_{n}\right) \bar{h} \tilde{f}_{t}^{x}=-D \bar{\gamma} f_{\mathrm{BCS}} e^{-i \operatorname{sign}(x) \frac{\varphi}{2}}$,

$$
\begin{aligned}
& D\left[\partial_{x}^{2} \tilde{f}_{t}^{a}+2 \overline{\mathcal{C}}_{x}^{a b}\left(\partial_{x} \tilde{f}_{t}^{b}\right)\right]-2\left|\omega_{n}\right| \tilde{f}_{t}^{a}-D \bar{\Gamma}^{a b} \tilde{f}_{t}^{b} \\
& \quad=\delta_{x, a} 2 i \operatorname{sign}\left(\omega_{n}\right) \bar{h} \tilde{f}_{s},
\end{aligned}
$$

where we introduced the Kronecker-Delta $\delta_{i, j}$, the components of the averaged spin precession tensor

$$
\overline{\mathcal{C}}_{k}^{a b}=\varepsilon^{a c b} \mathcal{A}_{k}^{c} d /(W+d)
$$

and averaged Dyakonov-Perell (DP) spin relaxation tensor

$$
\bar{\Gamma}^{a b}=\left(\mathcal{A}_{k}^{c} \mathcal{A}_{k}^{c} \delta_{a, b}-\mathcal{A}_{k}^{a} \mathcal{A}_{k}^{b}\right) d /(W+d) .
$$

The averaged coupling constants are defined as $\bar{h}^{a}=$ $h^{a} W /(W+d), \quad \bar{\alpha}=\alpha d /(W+d), \quad \bar{\beta}=\beta d /(W+d), \quad$ and $\bar{\gamma}=\gamma /(W+d)$. The spatial dependence of the SOC fields, exchange field, and order parameter in the $x$ direction is not explicitly written and is defined in Eqs. (6), (7), and (9). In the rotated system the nonvanishing spin precession tensor elements are

$$
\begin{gathered}
\overline{\mathcal{C}}_{x}^{x z}=-\overline{\mathcal{C}}_{x}^{z x}=-\bar{\alpha} \cos (\vartheta)-\bar{\beta} \sin (\vartheta), \\
\overline{\mathcal{C}}_{x}^{y z}=-\overline{\mathcal{C}}_{x}^{z y}=\bar{\alpha} \sin (\vartheta)-\bar{\beta} \cos (\vartheta) .
\end{gathered}
$$

The nonzero elements of the DP spin relaxation tensor are

$$
\begin{gathered}
\bar{\Gamma}^{x x}(\vartheta)=\bar{\Gamma}^{y y}(-\vartheta)=\left(\bar{\alpha}^{2}+\bar{\beta}^{2}+\bar{\alpha} \bar{\beta} \sin (2 \vartheta)\right) \frac{W+d}{d} \\
\bar{\Gamma}^{z z}(\vartheta)=\bar{\Gamma}^{x x}(\vartheta)+\bar{\Gamma}^{y y}(\vartheta), \\
\bar{\Gamma}^{x y}(\vartheta)=\bar{\Gamma}^{y x}(\vartheta)=2 \bar{\alpha} \bar{\beta} \cos (2 \vartheta) \frac{W+d}{d} .
\end{gathered}
$$

The solution of Eqs. (12) and (13) and its covariant derivative are continuous at the boundaries $x= \pm L / 2$ between the different regions thus:

$$
\begin{gathered}
\left.\partial_{x} \tilde{f}_{s}\right|_{x= \pm \frac{L}{2}+0^{-}}=\left.\partial_{x} \tilde{f}_{s}\right|_{x= \pm \frac{L}{2}+0^{+}} \\
\left.\partial_{x} \tilde{f}_{t}^{a}\right|_{x= \pm \frac{L}{2}+0^{\mp}}=\left[\partial_{x} \tilde{f}_{t}^{a}+\overline{\mathcal{C}}_{x}^{a b} \tilde{f}_{t}^{b}\right]_{x= \pm \frac{L}{2}+0^{ \pm}} .
\end{gathered}
$$

The Eqs. (12) and (13) together with the boundary conditions Eqs. (21) and (22) fully determine the condensate within the limits of the mentioned approximations. Finally the current in 
the bridge region is given by

$$
j=4 \pi e N_{0} D T \sum_{\omega_{n}} \operatorname{Im}\left[\tilde{f}_{s}^{*} \partial_{x} \tilde{f}_{s}-\left(\tilde{f}_{t}^{i}\right)^{*}\left(\partial_{x} \tilde{f}_{t}^{i}\right)\right] .
$$

\section{B. Usadel equations for type 2 lateral junction}

For the junction type 2 the SOC coupling and the exchange field are finite over the whole bridge. Consequently $\bar{h}^{a}=h$, $\bar{\alpha}=\alpha, \bar{\beta}=\beta$, and $\bar{\gamma}=\gamma / W$. Thus the $z$-integrated Usadel equation is like in Eqs. (12) and (13) where now

$$
\overline{\mathcal{C}}_{k}^{a b}=\mathcal{C}_{k}^{a b}=\varepsilon^{a c b} \mathcal{A}_{k}^{c}
$$

and the DP spin relaxation tensor

$$
\bar{\Gamma}^{a b}=\Gamma^{a b}=\mathcal{A}_{k}^{c} \mathcal{A}_{k}^{c} \delta_{a, b}-\mathcal{A}_{k}^{a} \mathcal{A}_{k}^{b} .
$$

The spatial dependence of the SOC fields is now given by Eq. (8). The solution of this system of equations is continuous and fulfills

$$
\begin{gathered}
\left.\partial_{x} \tilde{f}_{s}\right|_{x= \pm \frac{L}{2}+0^{-}}=\left.\partial_{x} \tilde{f}_{s}\right|_{x= \pm \pm \frac{L}{2}+0^{+}}, \\
{\left[\partial_{x} \tilde{f}_{t}^{a}+\mathcal{C}_{x}^{a b} \tilde{f}_{t}^{b}\right]_{x= \pm \frac{L}{2}+0^{\mp}}=\left.\partial_{x} \tilde{f}_{t}^{a}\right|_{x= \pm \frac{L}{2}+0^{ \pm}} .}
\end{gathered}
$$

Thus for type 2 junctions, the condensate function is determined from Eqs. (12) and (13) and Eqs. (26) and (27). Finally the current through the junction is given by

$$
\begin{aligned}
j= & 4 \pi e N_{0} D T \sum_{\omega_{n}} \operatorname{Im}\left[\tilde{f}_{s}^{*} \partial_{x} \tilde{f}_{s}-\left(\tilde{f}_{t}^{i}\right)^{*}\left(\partial_{x} \tilde{f}_{t}^{i}\right)\right. \\
& \left.+\left(\tilde{f}_{t}^{z}\right)^{*}\left(\alpha \tilde{f}_{t}^{x}+\beta \tilde{f}_{t}^{y}\right)\right] .
\end{aligned}
$$

\section{THE JOSEPHSON CURRENT IN TYPE 1 JUNCTIONS: ANALYTICAL SOLUTION}

Here we focus on type 1 junctions in the case when the exchange interaction is the dominant energy scale, $D \alpha^{2}, D \beta^{2}, D \alpha \beta, T \ll h$. The junction is larger than the magnetic length, $\xi_{h}$, and hence the current is solely determined by the LRTC, $\tilde{f}_{t}^{y}$ and $\tilde{f}_{t}^{z}$. The other two components decay over $\xi_{h}$ in the $\mathrm{F}$ region.

We solve Eqs. (12) and (13) perturbatively up to second order in the SOC fields, $\mathcal{A}_{k}^{a}$. In zeroth order only the singlet and triplet component parallel to the field, $\tilde{f}_{t, 0}^{x}$, are finite. Their explicit form is given in the Appendix, Eq. (A13). In first order in the SOC the component $\tilde{f}_{t}^{z}$ appears as a consequence of the precession term. Specifically it is determined by

$$
\partial_{x}^{2} \tilde{f}_{t, 1}^{z}-\frac{2\left|\omega_{n}\right|}{D} \tilde{f}_{t, 1}^{z}=-2 \overline{\mathcal{C}}_{x}^{z x}\left(\partial_{x} \tilde{f}_{t, 0}^{x}\right) .
$$

The component $\tilde{f}_{t}^{y}$ appears in second order of the SOC and satisfies:

$$
\partial_{x}^{2} \tilde{f}_{t, 2}^{y}-\frac{2\left|\omega_{n}\right|}{D} \tilde{f}_{t, 2}^{y}=-2 \overline{\mathcal{C}}_{x}^{y z}\left(\partial_{x} \tilde{f}_{t, 1}^{z}\right)+\bar{\Gamma}_{y x}(\vartheta) \tilde{f}_{t, 0}^{x} .
$$

The explicit expressions for these components are given in the Appendix, Eqs. (A20), (A24). From these solutions we obtain the current density in the $\mathrm{F}$ region. The current density Eq. (5) is only due to the contribution of the long-range components $\tilde{f}_{t}^{z}$ and $\tilde{f}_{t}^{y}$ in Eq. (28). The maximum value of the Josephson current, i.e., the critical current $j_{c}$, is obtained at $\varphi=\pi / 2$ :

$$
\begin{aligned}
j_{c}= & j\left(\varphi=\frac{\pi}{2}\right)=\pi e N_{0} D T \sum_{\omega_{n}}\left|f_{x}^{b}\right|^{2} e^{-\kappa_{\omega} L} \\
& \times\left(\frac{(\bar{\alpha} \cos \vartheta+\bar{\beta} \sin \vartheta)^{2}}{2 \kappa_{\omega}}-\frac{8 \bar{\alpha}^{2} \bar{\beta}^{2} \cos ^{2} 2 \vartheta}{\kappa_{\omega}^{3}}\right)
\end{aligned}
$$

where we define $\kappa_{\omega}=\sqrt{2\left|\omega_{n}\right| / D}$ and

$$
f_{x}^{b} \approx-i \frac{\gamma \operatorname{sign}\left(\omega_{n}\right) \xi_{h}^{2}}{2} f_{\mathrm{BCS}}
$$

is the value of $\tilde{f}_{t}^{x}$ for zero SOC, in the $\mathrm{F}$ region below the superconducting electrodes far from the bridge region. The first term in the second line of Eq. (31) is the lowest correction in the SOC which stems from the precession term in Eq. (29) and generates the LRTC $\tilde{f}_{t}^{z}$ from rotation of the short-range $\tilde{f}_{t, 0}^{x}$. It is a positive contribution ( 0 junction) and as expected depends on the direction of the field. It vanishes for an angle $\vartheta_{0} \in[\pi / 2, \pi]$ that depends on the relative strength of the considered SOC types and is given by $\vartheta_{0}=\arctan \left(-\frac{\alpha}{\beta}\right)+$ $n \pi$ [cf. with the numerical results shown in Figs. 2(a)-2(c)].

In the next order of the SOC the contribution to the current is the negative, second term in the second line of Eq. (31), and it is due to the spin relaxation term $\Gamma_{y x}$ in Eq. (30), that leads to a finite $\tilde{f}_{t}^{y}$ component. This contribution is only finite if both Rashba and Dresselhaus type of SOC are present. This explains why in the case of a pure Rashba or Dresselhaus SOC the current does not change sign as a function of $\vartheta$ [see numerical results shown in Figs. 2(a), 2(b) 2(d), and 2(e)].

Thus, the sign and magnitude of the critical current is determined by two competing contributions, namely spin precession and anisotropic spin relaxation [11], which in turn depend strongly on the direction of the applied Zeeman field. For example the contribution due to spin precession is zero whenever the SU(2) electric field strength in transport direction $\mathcal{F}_{x, 0}(\vartheta)=-i\left[\hat{\mathcal{A}}_{x}, \hat{h}(\vartheta)\right]$ vanishes. This is in accordance with previous theoretical investigations that identified $\mathcal{F}_{k, 0}$ as the generator of the LRTC [10,11]. According to Eq. (31), $\mathcal{F}_{x, 0}\left(\vartheta_{0}\right)=0$. For this value of $\vartheta$ the second negative term dominates provided that $\cos 2 \vartheta_{0} \neq 0$ and leads to a change of sign of the critical current, a $0-\pi$ transition.

For either pure Rashba or pure Dresselhaus SOC the dependence of the critical current on $\vartheta$ is simply shifted by $\pi / 2$ for the same magnitude of the SOC parameter. This can be already inferred from Eq. (1), which is symmetric when interchanging $\alpha \leftrightarrow \beta$ and $x \leftrightarrow y$ for the coordinate labels in spin space.

Equation (31) is valid for a symmetric junction, i.e., a junction of type 1 with the same SOC at both electrodes. In the case that the left $(\mathrm{L})$ and right $(\mathrm{R})$ electrodes have different values for the Rashba and Dresselhaus SOCs $\alpha_{L / R}$ and $\beta_{L / R}$ it is possible to obtain a $0-\pi$ transition solely due to spin precession effects. Namely, the critical current up to first order in the SOC fields reads

$$
\begin{aligned}
j_{c}= & \pi e N_{0} D T \sum_{\omega_{n}} \frac{\left|f_{x}^{b}\right|^{2}}{\kappa_{\omega}} e^{-\kappa_{\omega} L} \\
& \times\left(\bar{\alpha}_{L} \cos \vartheta+\bar{\beta}_{L} \sin \vartheta\right)\left(\bar{\alpha}_{R} \cos \vartheta+\bar{\beta}_{R} \sin \vartheta\right) .
\end{aligned}
$$




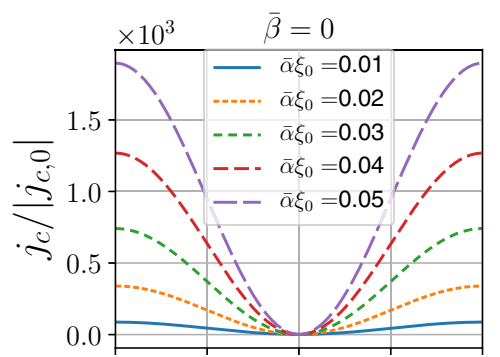

(a)

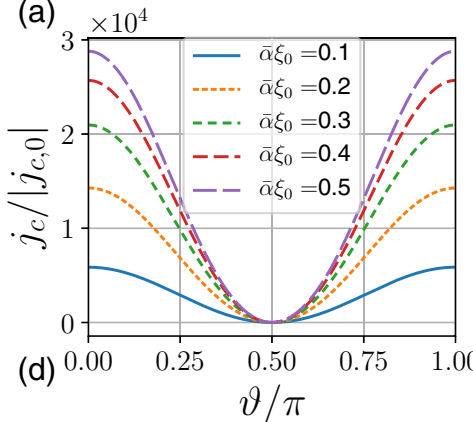

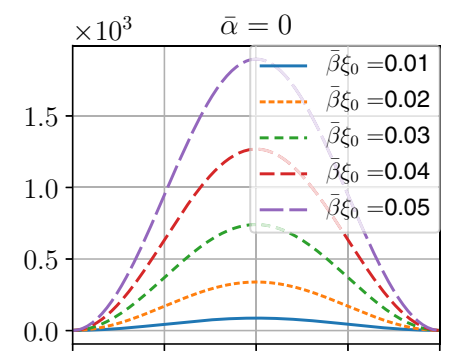

(b)

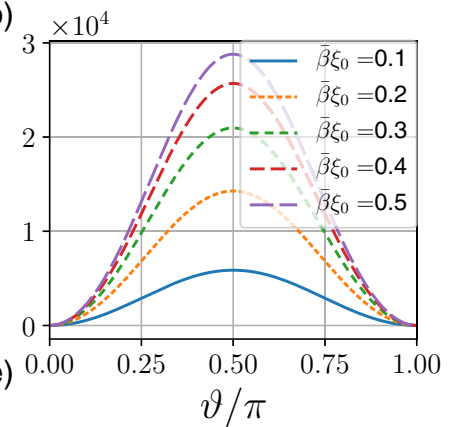

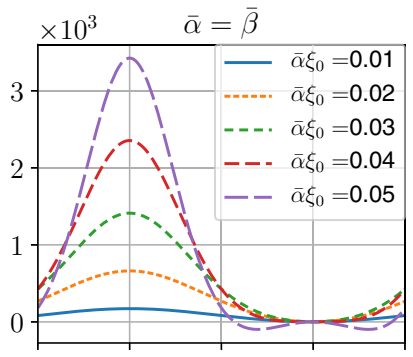

(c)

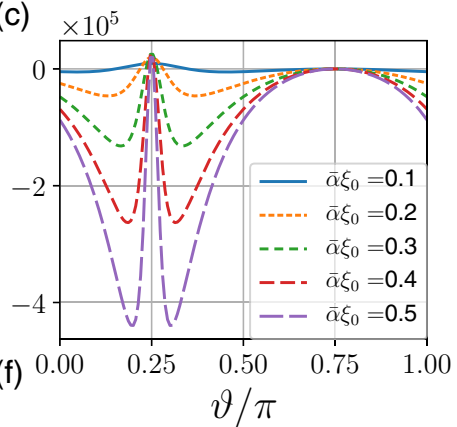

FIG. 2. Numerical results for the critical current as a function of the orientation of an in-plane exchange field for the type 1 junction. Different curves correspond to different values of the SOC parameters. In all plots we set $\bar{h}=10 \Delta, L=5 \xi_{0}, T=0.01 \Delta$ and the thickness of the SOC and $\mathrm{F}$ layer are chosen such that $d / W=1$.

By inspecting Eq. (33) we see that the current reversal appears every time the $S U(2)$ electric field strength disappears in the left or right lead $\mathcal{F}_{x, 0}^{L / R}=0$, as long as $\alpha_{L} \beta_{R} \neq \alpha_{R} \beta_{L}$. When all couplings are nonvanishing this takes place at the angles $\vartheta_{0}^{L / R}=\arctan \left(-\frac{\alpha_{L / R}}{\beta_{L / R}}\right)+n \pi$. The interval, where the current is reversed with respect to the symmetric case, is maximized when there is only Rashba SOC in one lead and only Dresselhaus SOC in the other as then $j_{c} \propto \alpha \beta \sin (2 \vartheta)$.

To summarize this section, for low SOC strength, the long-range supercurrent is mainly determined by the spin precession. If the $\mathrm{S}$ electrodes are symmetric and only one type of SOC is active, the current can be switched on and off by rotating the exchange field in the $x-y$ plane, but no $0-\pi$ transition takes place. A reversal of the current only appears if both SOC types are finite and originates in a competition of the spin precession and spin relaxation effects. A current reversal due to spin precession effects can only be achieved by choosing leads with different SOC parameters.

\section{NUMERICAL RESULTS}

In this section we compute numerically the Josephson current for both types of junctions with finite $S$ electrodes. The total length of the system is $L_{\text {tot }}=2 L_{S}+L$, where $L_{S}$ is the length of the $S$ electrode, and is set to $L_{\text {tot }}=10 L$. The systems of equations (12) and (13) are complemented by the boundary condition Eq. (4) at the outer interfaces:

$$
\left.\tilde{\nabla}_{x} \hat{f}\right|_{x= \pm L_{\text {tot }} / 2}=0 .
$$

The resulting critical current density for the junction type 1 is shown in Figs. 2(a)-2(f) and for junction type 2 in Fig. 3(a)-3(f). For low SOC strengths and any of the studied SOC types and junction types, the current vanishes when the SU(2) electric field strength vanishes in accordance with previous theories [10]. Indeed, the critical current for both setups and small SOC show qualitatively identical behavior [Figs. 2(a)-2(c) and 3(a)-3(c)], in very good agreement with the analytical result of Eq. (31). This implies that at the level of spin-precession effects both junctions behave similarly. As expected the critical current curves for the case of pure Rashba or Dresselhaus SOC are shifted by $\pi / 2$ when comparing curves of corresponding SOC strengths.

When increasing the SOC strength for junction type 1 we observe the competition between the two LRTC generating mechanisms. Comparing the upper and lower panels of Fig. 2 we see that the current changes sign at sufficiently large SOC strengths, only when both Rashba and Dresselhaus SOC are finite, as expected. For the special case when $\alpha=\beta$ and exchange field orientation $\vartheta=\pi / 4$ there is no $0-\pi$ transition possible as the spin relaxation contribution to the current vanishes. At $\vartheta=3 / 4 \pi$ spin precession and spin relaxation contributions vanish simultaneously, as can be seen in Fig. 2(f).

By further increase of the SOC the numerical results shown in Fig. 2(f) differ qualitatively from the analytic ones: There is a strong increase of the critical current in two negative dips around $\vartheta=\pi / 4$. The two negative dips move closer to $\vartheta=\pi / 4$ by increasing the SOC strength. Also there is a flattening of the curve at $\vartheta=3 / 4 \pi$.

The $0-\pi$ transition due to spin precession effects in junction type 1 with asymmetric SOC obtained analytically in the previous section is confirmed by the numerics as shown in Fig. 4. In particular, the points of current reversal as a function of $\vartheta$ are in agreement with the analytical result, Eq. (33).

The case of large SOC in type 2 junctions is shown in Figs. 3(d)-3(f). We clearly see that $0-\pi$ transitions are possible for any choice of SOC. The case when $\alpha, \beta \neq 0$ is qualitatively similar to junction type 1 . In contrast, for 


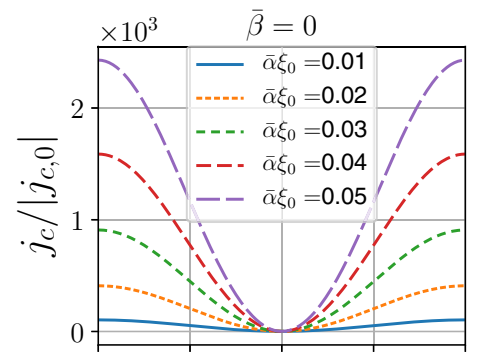

(a)

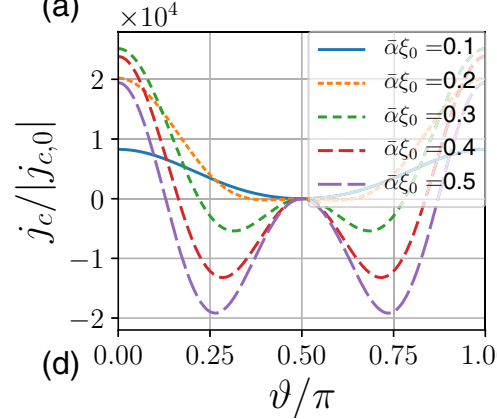

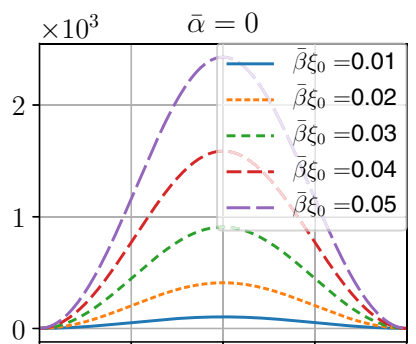

(b)

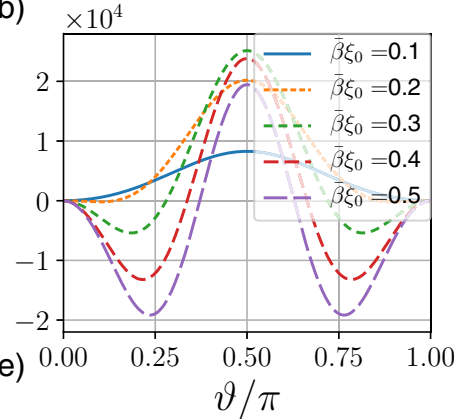

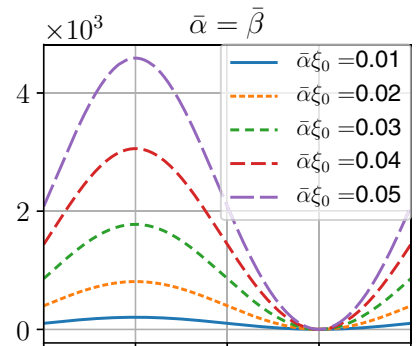

(c)

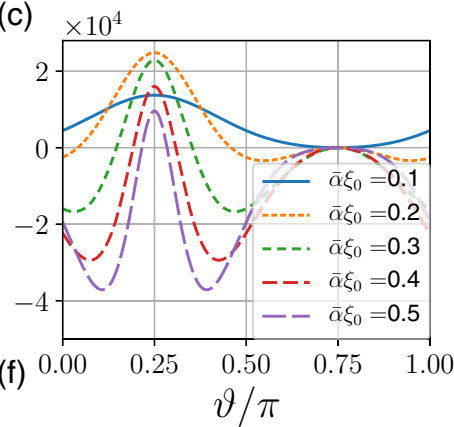

FIG. 3. Numerical results for the critical current as a function of the orientation of an in-plane exchange field for a type 2 junction. Different curves correspond to different values of the SOC parameters. In all plots we set $\bar{h}=10 \Delta, L=5 \xi_{0}, T=0.01 \Delta$.

junction $2,0-\pi$ transitions are possible for pure Rashba or Dresselhaus SOC when increasing the SOC strength, as shown in Figs. 3(c), 3(d), and 5. Our results, regarding the current sign reversal, are similar to the results of Ref. [27], where a one-dimensional junction with a pure Rashba has been studied. Similarly to the one-dimensional case, our results for two-dimensional SOC show that the direction of the current can be inverted by tuning the strength of the Rashba SOC, which can be done by a voltage gate if the bridge region is a semiconductor. Such a gate has also been suggested in Ref. [26] for creation of a long ranged spin-triplet helix in a ballistic ferromagnetic Josephson junction.

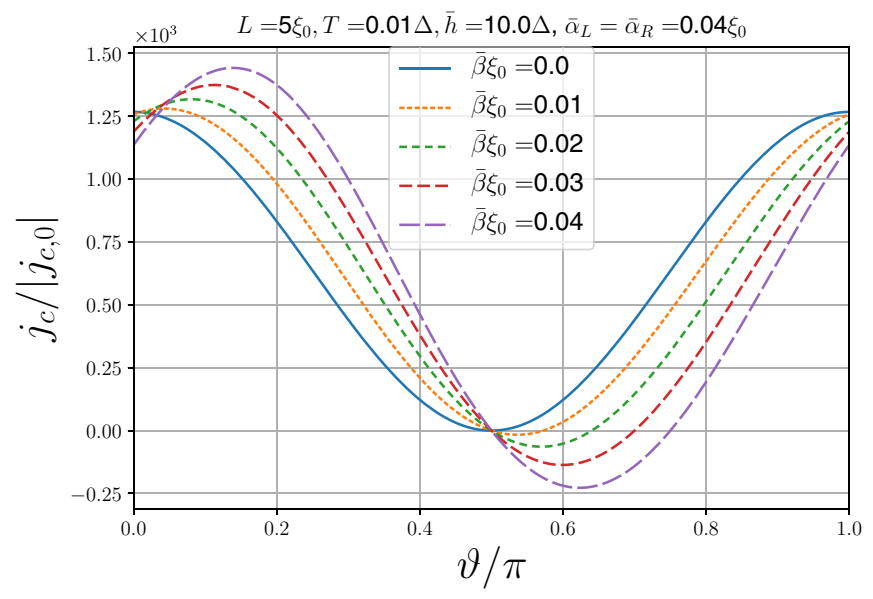

FIG. 4. Numerical results for the critical current as a function of the orientation of an in-plane exchange field for an asymmetric junction of type 1 . We set $\bar{h}=10 \Delta, L=5 \xi_{0}, T=0.01 \Delta$, and $d / W=1$.

\section{CONCLUSION}

We present a study of the effects of Rashba and Dresselhaus $\mathrm{SO}$ interaction in two types of diffusive lateral Josephson junctions. In the first type the bridge linking the superconducting electrodes is a ferromagnet and the SOC fields originated from heavy metal interlayers placed between the $\mathrm{S}$ leads and the $\mathrm{F}$ bridge. In the second geometry the exchange fields and SOC fields are finite over the whole bridge. In a realistic setup this can be realized by a $2 \mathrm{D}$ semiconducting bridge in an external magnetic field. In both cases we determine the long-range triplet Josephson current. We show how the magnitude and sign of the supercurrent can be controlled by varying the direction of the exchange field as well as tuning the strengths of the SOC. Besides their relevance for application as supercurrent valves such lateral junctions can be used as an unequivocal way of detecting the long-range triplet component of the condensate in lateral setups.

Note added: Recently, we became aware of the work Ref. [28] that studies junction type 1 in great detail. Our work confirms and extends the analytical and numerical results therein as the authors mainly focus on pure Rashba SOC.

\section{ACKNOWLEDGMENTS}

B.B. and F.S.B. acknowledge funding by the Spanish Ministerio de Ciencia, Innovación y Universidades (MICINN) under the project FIS2017-82804-P and by the Transnational Common Laboratory QuantumChemPhys. R.B. acknowledges funding from the European Union's Horizon 2020 research and innovation program under the Marie Skłodowska-Curie Grant Agreement No. 793318. 

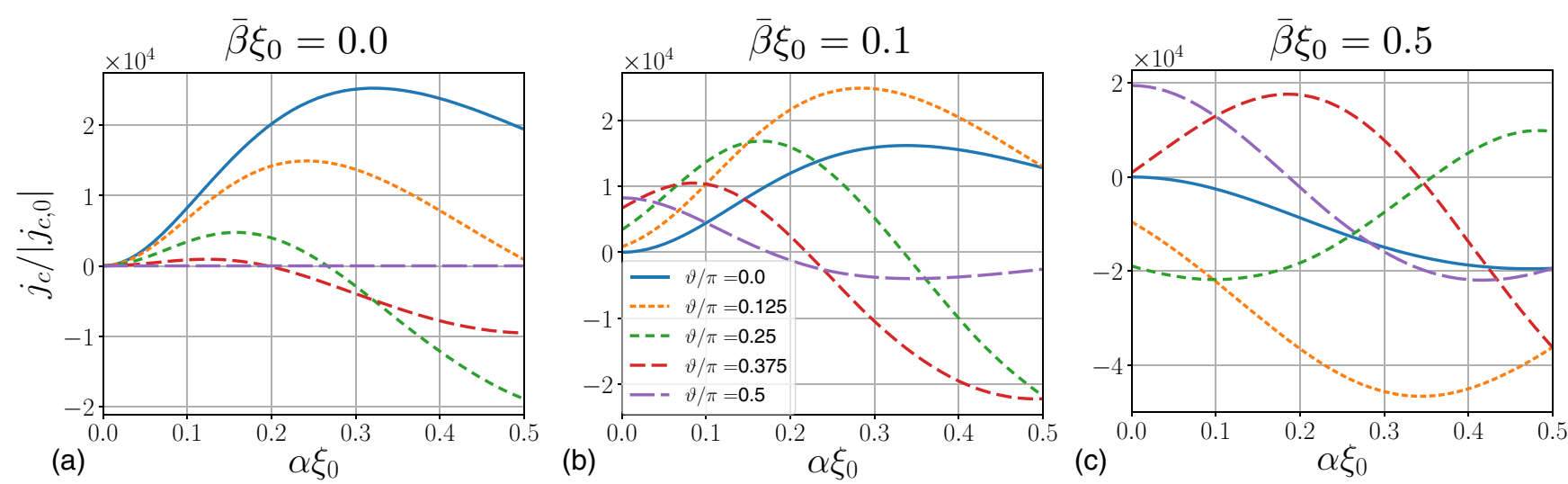

FIG. 5. Critical current for a junction of type 2 as function of the Rashba SOC strength $\alpha$. The Dresselhaus SOC parameter $\beta$ is increased from panel (a) to panel (c). Different curves correspond to different orientations of the exchange field. In all plots we set $\bar{h}=10 \Delta, L=5 \xi_{0}$, $T=0.01 \Delta$.

\section{APPENDIX: BASIC EQUATIONS}

After performing the $z$ integration, the resulting system of differential equations for the transformed anomalous Green's function $\hat{\tilde{f}}=\mathcal{U} \hat{f} \mathcal{U}^{\dagger}$ for $|x|>L / 2$ is:

$$
\begin{gathered}
D\left[\partial_{x}^{2} \tilde{f}_{s}\right]-2\left|\omega_{n}\right| \tilde{f}_{s}-2 i \operatorname{sign}\left(\omega_{n}\right) \bar{h} \tilde{f}_{t}^{x} \\
=-D \bar{\gamma} f_{\mathrm{BCS}} e^{-i \operatorname{sign}(x) \frac{\varphi}{2}} \\
D\left[\partial_{x}^{2} \tilde{f}_{t}^{x}+2 \overline{\mathcal{C}}_{x}^{x b}\left(\partial_{x} \tilde{f}_{t}^{b}\right)\right]-2\left|\omega_{n}\right| \tilde{f}_{t}^{x}-D \bar{\Gamma}^{x b} \tilde{f}_{t}^{b} \\
=2 i \operatorname{sign}\left(\omega_{n}\right) \tilde{f}_{s} \bar{h} \\
D\left[\partial_{x}^{2} \tilde{f}_{t}^{y}+2 \overline{\mathcal{C}}_{x}^{y b}\left(\partial_{x} \tilde{f}_{t}^{b}\right)\right]-2\left|\omega_{n}\right| \tilde{f}_{t}^{y}-D \bar{\Gamma}^{y b} \tilde{f}_{t}^{b}=0 \\
D\left[\partial_{x}^{2} \tilde{f}_{t}^{z}+2 \overline{\mathcal{C}}_{x}^{z b}\left(\partial_{x} \tilde{f}_{t}^{b}\right)\right]-2\left|\omega_{n}\right| \tilde{f}_{t}^{z}-D \bar{\Gamma}^{z b} \tilde{f}_{t}^{b}=0 .
\end{gathered}
$$

In the barrier region $|x|<L / 2$ we get

$$
\begin{gathered}
D \partial_{x}^{2} \tilde{f}_{s}-2\left|\omega_{n}\right| \tilde{f}_{s}-2 i \operatorname{sign}\left(\omega_{n}\right) \bar{h} \tilde{f}_{t}^{x}=0 \\
D \partial_{x}^{2} \tilde{f}_{t}^{x}-2\left|\omega_{n}\right| \tilde{f}_{t}^{x}-2 i \operatorname{sign}\left(\omega_{n}\right) \bar{h} \tilde{f}_{s}=0 \\
D \partial_{x}^{2} \tilde{f}_{t}^{y}-2\left|\omega_{n}\right| \tilde{f}_{t}^{y}=0 \\
D \partial_{x}^{2} \tilde{f}_{t}^{z}-2\left|\omega_{n}\right| \tilde{f}_{t}^{z}=0 .
\end{gathered}
$$

The $z$ integration causes an averaging of the couplings as described in the main text. The solution of this system of equations is continuous and fulfills

$$
\begin{gathered}
\left.\partial_{x} \tilde{f}_{s}\right|_{x= \pm \frac{L}{2}+0^{-}}=\left.\partial_{x} \tilde{f}_{s}\right|_{x= \pm \frac{L}{2}+0^{+}} \\
\left.\partial_{x} \tilde{f}_{t}^{a}\right|_{x= \pm \frac{L}{2}+0^{\mp}}=\left[\partial_{x} \tilde{f}_{t}^{a}+\overline{\mathcal{C}}_{x}^{a b} \tilde{f}_{t}^{b}\right]_{x= \pm \frac{L}{2}+0^{ \pm}},
\end{gathered}
$$

at the boundaries between the different regions. The spin precession tensor components $\mathcal{C}_{k}^{a b}$ and DP tensor components $\Gamma^{a b}$ in the rotated system are determined from the transformed fields

$$
\begin{gathered}
\hat{\tilde{\mathcal{A}}}_{x}=\frac{\hat{\sigma}^{x}}{2} \zeta(\vartheta)-\eta(\vartheta) \frac{\hat{\sigma}^{y}}{2}, \\
\hat{\tilde{\mathcal{A}}}_{y}=\frac{\hat{\sigma}^{x}}{2} \eta(-\vartheta)-\zeta(-\vartheta) \frac{\hat{\sigma}^{y}}{2},
\end{gathered}
$$

with $\eta(\vartheta)=\bar{\alpha} \cos (\vartheta)+\bar{\beta} \sin (\vartheta)$ and $\zeta(\vartheta)=-\bar{\alpha} \sin (\vartheta)+$ $\bar{\beta} \cos (\vartheta)$. The equations (A1)-(A10) fully determine the junction system within the limits of the approximations mentioned in the main text.

\section{Zeroth order correction}

As in the main text we consider the junction type 1 assuming semi-infinite leads. Solving the above system of equations for vanishing SOC gives the following zeroth order solution for the function $\tilde{f}_{t}^{x}$,

$$
\begin{aligned}
& \tilde{f}_{t, 0}^{x} \\
& \quad=\left\{\begin{array}{l}
\frac{A_{1}^{L}}{\lambda^{+}} e^{\lambda^{+} x}+\frac{A_{2}^{L}}{\lambda^{-}} e^{\lambda^{-} x}+f_{x}^{b} e^{-i \frac{\varphi}{2}}, x<-\frac{L}{2} \\
\frac{B_{1}}{\lambda^{+}} e^{\lambda^{+} x}-\frac{B_{2}}{\lambda^{+}} e^{-\lambda^{+} x}+\frac{B_{3}}{\lambda^{-}} e^{\lambda^{-} x}-\frac{B_{4}}{\lambda^{-}} e^{-\lambda^{-} x} \\
-\frac{A_{1}^{R}}{\lambda^{+}} e^{-\lambda^{+} x}-\frac{A_{2}^{R}}{\lambda^{-}} e^{-\lambda^{-} x}+f_{x}^{b} e^{i \frac{\varphi}{2}}, x>\frac{L}{2},
\end{array}\right.
\end{aligned}
$$

where $\lambda^{ \pm}=\sqrt{\frac{2\left|\omega_{n}\right|}{D} \pm i \frac{2 \operatorname{sgn}\left(\omega_{n}\right) \bar{h}}{D}}$,

$$
\begin{gathered}
A_{1 / 2}^{L}=\mp \lambda^{ \pm} \frac{f_{s}^{b} \pm f_{x}^{b}}{2} \sinh \left(\frac{L \lambda^{ \pm}-i \varphi}{2}\right) \\
B_{1 / 2}= \pm \frac{\lambda^{+}}{4}\left(f_{s}^{b}+f_{x}^{b}\right) \exp \left(\frac{-L \lambda^{+} \pm i \varphi}{2}\right) \\
B_{3 / 4}=\mp \frac{\lambda^{-}}{4}\left(f_{s}^{b}-f_{x}^{b}\right) \exp \left(\frac{-L \lambda^{-} \pm i \varphi}{2}\right) \\
A_{1 / 2}^{R}= \pm \lambda^{ \pm} \frac{f_{s}^{b} \pm f_{x}^{b}}{2} \sinh \left(\frac{L \lambda^{ \pm}+i \varphi}{2}\right)
\end{gathered}
$$

and the bulk solutions for the singlet and triplet $x$ component

$$
\begin{gathered}
f_{s}^{b}=D \gamma \frac{f_{\mathrm{BCS}}}{2} \frac{\left|\omega_{n}\right|}{\left|\omega_{n}\right|^{2}+h^{2}} \approx \frac{\gamma\left|\omega_{n}\right| \xi_{\tilde{h}}^{2}}{2 \bar{h}} f_{\mathrm{BCS}} \\
f_{x}^{b}=-i D \gamma \frac{f_{\mathrm{BCS}}}{2} \frac{\operatorname{sign}\left(\omega_{n}\right) \bar{h}}{\left|\omega_{n}\right|^{2}+\bar{h}^{2}} \approx-i \frac{\gamma \operatorname{sign}\left(\omega_{n}\right) \xi_{\bar{h}}^{2}}{2} f_{\mathrm{BCS}} \\
\text { with } \xi_{\bar{h}}=\sqrt{D / \bar{h}} .
\end{gathered}
$$




\section{First order correction}

The ansatz for the solution of Eq. (29) reads

$$
\tilde{f}_{t, 1}^{z}(x)= \begin{cases}K_{1} e^{\kappa_{\omega} x}+Z_{1}^{L} e^{\lambda^{+} x}+Z_{2}^{L} e^{\lambda^{-} x}, & x<-\frac{L}{2} \\ K_{2} e^{\kappa_{\omega} x}+K_{3} e^{-\kappa_{\omega} x}, & |x|<\frac{L}{2} \\ K_{4} e^{-\kappa_{\omega} x}+Z_{1}^{R} e^{-\lambda^{+} x}+Z_{2}^{R} e^{-\lambda^{-} x}, & x>\frac{L}{2}\end{cases}
$$

where

$$
\begin{aligned}
& Z_{1}^{L}=-\frac{\eta(\vartheta) A_{1}^{L}}{\left(\lambda^{+2}-\kappa_{\omega}^{2}\right)}, \quad Z_{2}^{L}=-\frac{\eta(\vartheta) A_{2}^{L}}{\left(\lambda^{-2}-\kappa_{\omega}^{2}\right)}, \\
& Z_{1}^{R}=-\frac{\eta(\vartheta) A_{1}^{R}}{\left(\lambda^{+2}-\kappa_{\omega}^{2}\right)}, \quad Z_{2}^{R}=-\frac{\eta(\vartheta) A_{2}^{R}}{\left(\lambda^{-2}-\kappa_{\omega}^{2}\right)} .
\end{aligned}
$$

Keeping only leading order terms when $\bar{h} \gg T, \max \left\{\bar{\Gamma}^{a b}\right\}$ and assuming $L \gg \xi_{\bar{h}}$ we find

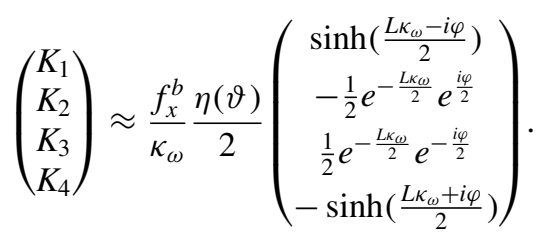

\section{Second order correction}

The ansatz for the solution of Eq. (30) reads

$$
\tilde{f}_{t, 2}^{y}(x)=\left\{\begin{array}{l}
\left(L_{1}+x Y_{1}^{L}\right) e^{\kappa_{\omega} x}+Y_{2}^{L} e^{\lambda^{+} x}+Y_{3}^{L} e^{\lambda^{-} x}+Y_{4}^{L}, x<-\frac{L}{2} \\
L_{2} e^{\kappa_{\omega} x}+L_{3} e^{-\kappa_{\omega} x},|x|<\frac{L}{2} \\
\left(L_{4}+x Y_{1}^{R}\right) e^{-\kappa_{\omega} x}+Y_{2}^{R} e^{-\lambda^{+} x}+Y_{3}^{R} e^{-\lambda^{-} x}+Y_{4}^{R}, x>\frac{L}{2}
\end{array}\right.
$$

with

$$
\begin{gathered}
Y_{1}^{L / R}=\zeta(\vartheta) K_{1 / 4}, \\
Y_{2}^{L / R}= \pm \frac{2 \lambda^{+2} \zeta(\vartheta) Z_{1}^{L}+\bar{\Gamma}_{y x} A_{1}^{L / R}}{\lambda^{+}\left(\lambda^{+2}-\kappa_{\omega}^{2}\right)}, \\
Y_{3}^{L / R}= \pm \frac{2 \lambda^{-2} \zeta(\vartheta) Z_{2}^{L}+\bar{\Gamma}_{y x} A_{2}^{L / R}}{\lambda^{-}\left(\lambda^{-2}-\kappa_{\omega}^{2}\right)}, \\
Y_{4}^{L / R}=-\bar{\Gamma}_{y x} \frac{f_{x}^{b} e^{\mp i \frac{\varphi}{2}}}{\kappa_{\omega}^{2}} .
\end{gathered}
$$

Considering only leading order terms when $\bar{h} \gg T, \max \left\{\bar{\Gamma}^{a b}\right\}$ consistent with the first order correction and assuming that $L \gg \xi_{\bar{h}}$ gives for the relevant coefficients inside the bridge

$$
\left(\begin{array}{l}
L_{2} \\
L_{3}
\end{array}\right)=-\frac{1}{2} e^{-\frac{L K}{2}}\left(\begin{array}{l}
Y_{4}^{R} \\
Y_{4}^{L}
\end{array}\right)
$$

[1] F. S. Bergeret, A. F. Volkov, and K. B. Efetov, Phys. Rev. Lett. 86, 4096 (2001).

[2] F. S. Bergeret, A. F. Volkov, and K. B. Efetov, Rev. Mod. Phys. 77, 1321 (2005).

[3] M. Eschrig, Phys. Today 64(1), 43 (2011); Rep. Prog. Phys. 78, 104501 (2015).

[4] J. Linder and J. W. Robinson, Nat. Phys. 11, 307 (2015).

[5] L. N. Bulaevskii, V. V. Kuzii, and A. A. Sobyanin, Pis'ma Zh. Éksp. Teor. Fiz. 25, 314 (1977) [JETP Lett. 25, 290 (1977)].
[6] A. I. Buzdin, L. N. Bulaevskil, and S. V. Panyukov, Pis'ma Zh. Eksp. Teor. Fiz. 35, 147 (1982) [JETP Lett. 35, 178 (1982)].

[7] V. V. Ryazanov, V. A. Oboznov, A. Y. Rusanov, A. V. Veretennikov, A. A. Golubov, and J. Aarts, Phys. Rev. Lett. 86, 2427 (2001).

[8] F. S. Bergeret, A. F. Volkov, and K. B. Efetov, Phys. Rev. B 64, 134506 (2001).

[9] T. Kontos, M. Aprili, J. Lesueur, F. Genêt, B. Stephanidis, and R. Boursier, Phys. Rev. Lett. 89, 137007 (2002). 
[10] F. S. Bergeret and I. V. Tokatly, Phys. Rev. Lett. 110, 117003 (2013).

[11] F. S. Bergeret and I. V. Tokatly, Phys. Rev. B 89, 134517 (2014).

[12] J. Robinson, J. Witt, and M. Blamire, Science 329, 59 (2010).

[13] M. S. Anwar, F. Czeschka, M. Hesselberth, M. Porcu, and J. Aarts, Phys. Rev. B 82, 100501(R) (2010).

[14] X. L. Wang, A. Di Bernardo, N. Banerjee, A. Wells, F. S. Bergeret, M. G. Blamire, and J. W. A. Robinson, Phys. Rev. B 89, 140508(R) (2014).

[15] E. C. Gingrich, P. Quarterman, Y. Wang, R. Loloee, W. P. Pratt, and N. O. Birge, Phys. Rev. B 86, 224506 (2012).

[16] J. W. A. Robinson, F. Chiodi, M. Egilmez, G. B. Halász, and M. G. Blamire, Sci. Rep. 2, 699 (2012).

[17] F. Chiodi, J. D. S. Witt, R. G. J. Smits, L. Qu, G. B. Halász, C.-T. Wu, O. T. Valls, K. Halterman, J. W. A. Robinson, and M. G. Blamire, Europhys. Lett. 101, 37002 (2013).

[18] A. Pal, Z. Barber, J. Robinson, and M. Blamire, Nat. Commun. 5, 3340 (2014).

[19] J. W. A. Robinson, N. Banerjee, and M. G. Blamire, Phys. Rev. B 89, 104505 (2014).

[20] Y. Kalcheim, O. Millo, M. Egilmez, J. W. A. Robinson, and M. G. Blamire, Phys. Rev. B 85, 104504 (2012).
[21] N. Banerjee, C. B. Smiet, R. G. J. Smits, A. Ozaeta, F. S. Bergeret, M. G. Blamire, and J. W. A. Robinson, Nat. Commun. 5, 3048 (2014).

[22] T. S. Khaire, M. A. Khasawneh, W. P. Pratt, and N. O. Birge, Phys. Rev. Lett. 104, 137002 (2010).

[23] N. Satchell and N. O. Birge, Phys. Rev. B 97, 214509 (2018).

[24] N. Banerjee, J. A. Ouassou, Y. Zhu, N. A. Stelmashenko, J. Linder, and M. G. Blamire, Phys. Rev. B 97, 184521 (2018).

[25] N. Satchell, R. Loloee, and N. O. Birge, Phys. Rev. B 99, 174519 (2019).

[26] X. Liu, J. K. Jain, and C.-X. Liu, Phys. Rev. Lett. 113, 227002 (2014).

[27] J. Arjoranta and T. T. Heikkilä, Phys. Rev. B 93, 024522 (2016).

[28] J. R. Eskilt, M. Amundsen, N. Banerjee, and J. Linder, Phys. Rev. B 100, 224519 (2019).

[29] K. Usadel, Phys. Rev. Lett. 25, 507 (1970).

[30] Equation (1) is written in the strict diffusive limit and does not take into account charge-spin conversion terms which are higher order in the momentum relaxation rate $[32,33]$.

[31] M. Y. Kupriyanov and V. F. Lukichev, Zh. Éksp. Teor. Fiz. 94, 139 (1988) [Sov. Phys. JETP 67, 1163 (1988)].

[32] F. Konschelle, I. V. Tokatly, and F. S. Bergeret, Phys. Rev. B 92 , 125443 (2015).

[33] F. S. Bergeret and I. V. Tokatly, EPL 110, 57005 (2015). 